POS $\quad$ PROCEEDINGS

\title{
Neutrino-driven winds and nucleosynthesis
}

\author{
A. Arcones ${ }^{* \dagger}$ \\ Institut für Kernphysik, TU Darmstadt, Schlossgartenstr. 9, D-64289 Darmstadt, Germany \\ Gesellschaft für Schwerionenforschung, Planckstr. 1, D-64291 Darmstadt, Germany \\ E-mail: a.arconesegsi.de \\ G. Martínez-Pinedo \\ Gesellschaft für Schwerionenforschung, Planckstr. 1, D-64291 Darmstadt, Germany
}

\section{H.-Th. Janka}

Max-Planck-Institut für Astrophysik, Karl-Schwarzschild-Str. 1, D-85741 Garching, Germany

\section{A. Schwenk}

TRIUMF, 4004 Wesbrook Mall, Vancouver, BC,V6T 2A3, Canada

\section{E. O'Connor}

TRIUMF, 4004 Wesbrook Mall, Vancouver, BC,V6T 2A3, Canada

Department of Physics, California Institute of Technology, Pasadena, CA 91125

\section{K. Langanke}

Institut für Kernphysik, TU Darmstadt, Schlossgartenstr. 9, D-64289 Darmstadt, Germany Gesellschaft für Schwerionenforschung, Planckstr. 1, D-64291 Darmstadt, Germany

\section{C.J. Horowitz}

Nuclear Theory Center and Department of Physics, Indiana University, Bloomington, IN 47408

\begin{abstract}
Baryonic outflows from proto-neutron stars formed in core-collapse supernova explosions are one of the possible scenarios for the production of heavy elements via the r-process. If the ejected matter reaches supersonic velocities the outflow is known as neutrino-driven wind. We have studied the long-time evolution of proto-neutron stars with one- and two-dimensional hydrodynamical simulations and performed nucleosynthesis calculations with the resulting wind trajectories. We find that the present wind models are much closer than previous studies to provide suitable conditions for r-process nucleosynthesis. Moreover, we explored the effect of the wind terminations shock, nuclear physics input, and composition of the outer layers of the neutron star on the nucleosynthesis production.
\end{abstract}

10th Symposium on Nuclei in the Cosmos

July 27 - August 12008

Mackinac Island, Michigan, USA

\footnotetext{
*Speaker.

${ }^{\dagger}$ supported by the Deutsche Forschungsgemeinschaft through contract SFB 634
} 


\section{Introduction}

The question of whether heavy elements are produced in supernova explosion is still open despite the numerous works done in the last 50 years (see [2] for a review). The requirements for the r-process to take place in the neutrino-driven wind developing after a core-collapse supernova explosion are ([3]): high entropies $(\mathrm{S} \approx 200)$, electron fraction $\mathrm{Ye}<0.5$, and fast expansion (expansion time scale $\approx 2-10 \mathrm{~ms}$ ).

We analyzed hydrodynamical simulations of explosions and the evolution of the subsequent outflows ([1]) with the aim to understand which are the relevant ingredients for the r-process. We studied in a systematic way the nucleosynthesis production (in a post processing step) in this scenario and analyzed the influence of different factors (e.g. input nuclear physics).

Furthermore, we performed a detailed analysis of the composition of the outer layers of the protoneutron star and its influence on the neutrino spectra and, therefore, on the wind electron fraction, which is used as input in the nucleosynthesis calculations ([11]).

\section{Nucleosynthesis studies}

The neutrino-driven wind expands through the slow-moving matter that was ejected at the beginning of the explosion. When the supersonic outflow hits this matter a reverse shock forms. In order to study the effect of such a wind termination shock on the nucleosynthesis products, we have chosen trajectories from simulations of several different progenitor stars ([1]). The wind entropies from the simulations are too low and the electron fractions too large to produce heavy elements. Therefore, we varied artificially the initial electron fraction and the entropy and found that in order to form the third peak either rather small values of Ye around 0.2, or an increase of the entropy from around $100 \mathrm{k}_{\mathrm{B}} /$ nuc up to $200 \mathrm{k}_{\mathrm{B}} /$ nuc are required.

In order to evaluate the influence of the wind termination shock we took one trajectory from simulations and increased the entropy by a factor of 2, without varying the expansion and electron fraction. Based on this trajectory (labeled as normal in Fig. 1) the position of the reverse shock is artificially changed (see also [7, 6]). Figure 1 shows the evolution of temperature and neutron density for the trajectory with the original reverse shock position from the simulation (black line) and also in the cases where the reverse shock is placed at a tempertarures of $\mathrm{T}=0.5 \mathrm{GK}$ (red line) and 1GK (blue line) or totally removed (green line). More details of the prescription used for the extrapolation of the trajectory data when the reverse shock is moved are given in [12].

Relevant times scales are shown in Fig. 2 to the right: beta decay with dashed line, $(\gamma, \mathrm{n})$ with dotted line and $(\mathrm{n}, \gamma)$ with solid line. One can see that when the reverse shock is at higher temperatures (blue curve) the evolution takes place under $(n, \gamma)-(\gamma, n)$ equilibrium (dotted and solid lines coincide) this is the classical r-process. While at lower temperatures, as it is the case of the original trajectory, there is a competition between neutron capture and beta decay, this alternative to the r-process was already pointed out by $[13,7]$. These variations in the evolution translates into different r-process paths and therefore differences in the final abundances. Moreover, the relevant nuclear physics input also changes. If the system evolves in $(n, \gamma)-(\gamma, n)$ equilibrium, then neutron capture rates are not that relevant, while neutron separation energies and beta decay rates have a 

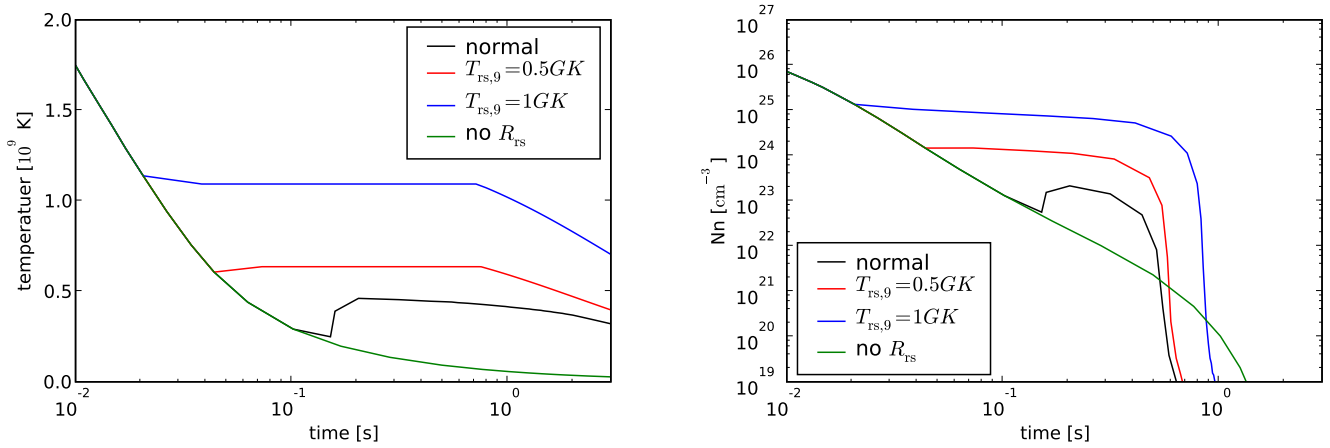

Figure 1: The left plot shows the temperature evolution of different trajectories analyzed with the network. The black line is the original trajectory of a $15 M_{\odot}$ progenitor star calculated by [1]. For the red and blue lines the position of the reverse shock has been moved to temperatures of 0.5 and $1 \mathrm{GK}$, respectively. And the green line is produced by removing the reverse shock and assuming an addiabatic expansion. The right figure is the corresponding neutron density evolution computed by the network (using FRDM for masses and neutron capture rates).

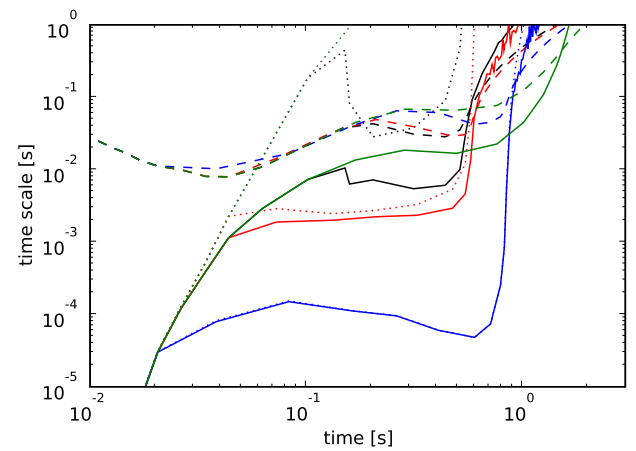

Figure 2: Representative time scales for the trajectories shown before with the same colors. Dashed lines are for beta decay rate, dotted lines $(\gamma, n)$, and solid line $(n, \gamma)$. When $(n, \gamma)-(\gamma, n)$ equilibrium is fulfil solid and dotted line coincide.

strong influence. On the other hand, if there is no equilibrium, then neutron captures and beta decays will determine the position of the peaks (Fig. 3).

To explore the sensitivity of the nuclear physics input we have repeated the nucleosynthesis calculations using two different sets of nuclear masses (FRDM and ETFSI-Q) and neutron capture rates calculated with them ([9]). The difference in the final abundace is shown in Fig. 3, where colored lines correspond to the trajectories shown in the left panel of Fig. 1. The left plot in Fig. 3 is calculated using FRDM, and the right one with ETFSI-Q, the influence of the nuclear physics input is clearly visible in the final abundances.

\section{Influence of light nuclei on neutrino-driven supernova outflows}

The thermodynamical conditions in the outer layers of protoneutron stars favor the presence of light nuclei (alpha particles, deuterons, tritons), which are not considered in the equations of state typically used in core-collapse supernova simulations. Using the profiles of the hydrodynamical 

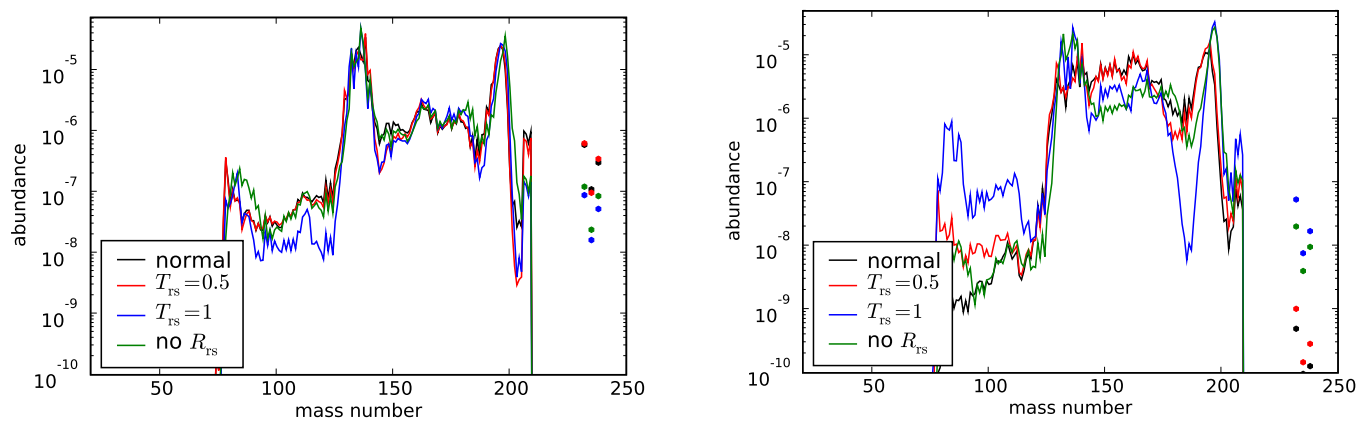

Figure 3: Abundance pattern for the trajectories shown with the same colors of previous figures. The left plot is calculated using FRDM for nuclear masses and neutron captures, while in the right one ETFSI-Q was employed.

simulations of [1], we estimated the effects of light nuclei on the emission of electron neutrinos and antineutrinos by means of virial ([8]) and NSE (nuclear statistical equilibrium) equations of state ([11]).

The appearance of light nuclei has only a minor impact on the average energy of the emitted electron neutrinos, but is significant for the average energy of antineutrinos. During the early post-explosion phase, the average energy of antineutrinos is slighly increased, while at later times during the protoneutron star cooling its reduced by about $1 \mathrm{MeV}$ (see table in [11]). The changes in the antineutrino average energies can have consequences for nucleosynthesis occuring in the neutrino-driven winds.

Left panel in Fig. 4 shows mass fractions for nuclei present around the surface of the protoneutron star using three different equations of state: the one of [1] (dashed lines), NSE EOS (dotted lines) and virial EoS (solid lines). The vertical lines mark the positions of the neutrinosphere of electron neutrinos (right line) and antineutrinos (left line). The neutrinosphere radius is defined as the position where the optical depth reaches $2 / 3$.

In the case of electron neutrinos the main contribution to the opacity is the absorption on neutrons. For antineutrinos, absorption on protons competes with other process as is shown in Fig. 4 right panel, where dashed lines are computed using the EOS from Arcones et al. (2007) (where no light nuclei are included) and solid lines using the virial EOS. The vertical lines mark the positions of the neutrinospheres of electron antineutrions for the two different EoS.

Our results suggest that future simulations of neutrino-driven supernova outflows should take into account light elements in the composition and their interactions with neutrinos.

\section{Conclusions}

We have studied different ingredients of the neutrino-driven wind in order to understand how reliable is this scenario as the site for the r-process. We have seen that including light elements to the composition used by the current supernova models has a direct effect on the outer layers of the neutron star and influences the neutrino spectra and therefore the wind electron fraction ([11]). Moreover, trajectories from long time supernova simulations ([1]) have been used as input 

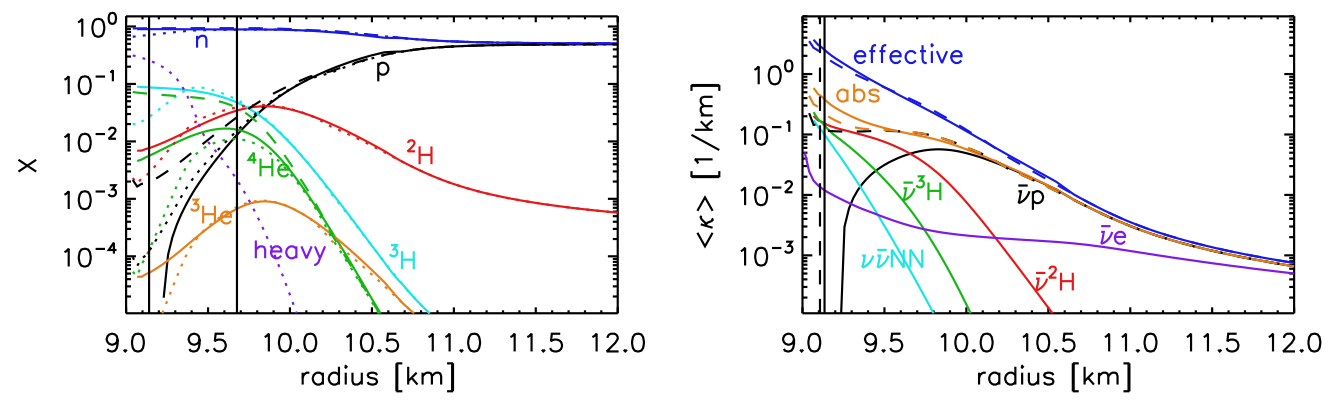

Figure 4: Mass fractions (left figure) for nuclei present around the surface of the protoneutron star using three different EOS (see text). The right figure shows different contributions to the absorption opacity and effective opacity of electron antineutrinos as a function of radius for two different EOS.

in a nuclear reaction network. Under the conditions found (too low entropy and too high electron fraction) in such neutrino-driven wind models there are not enough free neutrons to produce heavy elements. However, by modifying slightly the entropy it is possible to produce the third peak. This is an useful tool to analyze which ingredients of the nuclear physics input play an important role and under which conditions, as well as to understand which is the effect of the wind termination shock.

\section{References}

[1] A. Arcones, H.-Th. Janka, L. Scheck, AA 407, 1227 (2007)

[2] M. Arnould, S. Goriely, K. Takahashi, Phys.Rep 405, 97 (2007)

[3] Y.-Z. Qian, S. E.Woosley, ApJ 471, 331 (1996)

[4] T. A. Thompson, A. Burrows, B. S. Meyer, ApJ 562, 887 (2001)

[5] K. Otsuki and H. Tagoshi and T. Kajino and S. Wanajo, ApJ 533, 424 (2000)

[6] T. Kuroda, S. Wanajo, K. Nomoto, ApJ 672, 1068 (2008)

[7] S. Wanajo, ApJL, 666 L77 (2007)

[8] C. J. Horowitz, A. Schwenk, Nucl. Phys. A, 7755 (2006)

[9] T. Rauscher, F. K. Thielemann, At. Data Nucl. Data Tables,,75 1-352 (2000)

[10] K. Sumiyoshi, G. Röke Phys. Rev. C 77, 055804 (2008)

[11] A. Arcones, G. Martínez-Pinedo, O'Connor, A. Schwenk, H.-Th. Janka, C. J. Horowitz, K. Langanke, Phys. Rev. C 78, 015806 (2008)

[12] A. Arcones, G. Martínez-Pinedo, H.-Th. Janka, in prep.,

[13] J. B. Blake, D. N. Schramm, ApJ 209, 846 (1976) 